\title{
Decoupling Polarization, Crystal Tilt and Symmetry in Epitaxially-Strained Ferroelectric Thin Films Using 4D-STEM
}

\author{
Yu-Tsun Shao ${ }^{1}$, Zhen Chen ${ }^{1}$, Antonio Mei $^{2}$, Megan Holtz ${ }^{2}$, Elliot Padgett ${ }^{1}$, Darrell Schlom ${ }^{2,3}$ and David \\ A. Muller ${ }^{1,3 *}$ \\ 1. School of Applied and Engineering Physics, Cornell University, Ithaca, NY, USA. \\ 2. Department of Materials Science and Engineering, Cornell University, Ithaca, NY, USA. \\ 3. Kavli Institute at Cornell for Nanoscale Science, Ithaca, NY, USA. \\ * Corresponding author:dm24@cornell.edu
}

The mechanical and electrostatic boundary conditions at interfaces strongly affect both the domain structure and phase instabilities in ferroelectric films [1]. The coupling of two or more order parameters can induce chirality, for example the recently reported polarization vortices in $\mathrm{PbTiO}_{3} / \mathrm{SrTiO}_{3}$ superlattices [2]. Local electric field or atomic displacements can be measured with differential phase contrast (DPC) or annular dark field (ADF) STEM imaging. However, a slight crystal mistilt may complicate and dominate the interpretation of DPC-/ADF-STEM images $[3,4]$. The crystal tilt is associated with the disinclination strain in ferroelastic materials, which is often observed at domain boundaries [5]. Here, we explore the topological ordering of a potential vortex phase in multiferroic $\mathrm{BiFeO}_{3}$ films, where both polarization and crystal tilts are expected, using 4D-STEM and a high-speed electron microscope pixel array detector (EMPAD) for collecting full CBED patterns [6].

$\mathrm{A}\left(\mathrm{BiFeO}_{3}\right)_{\mathrm{m}} /\left(\mathrm{TbScO}_{3}\right)_{\mathrm{n}}(\mathrm{m}=\mathrm{n}=13)$ superlattice is studied here, in which $\mathrm{m}$ and $\mathrm{n}$ refers to the thickness in each layer in unit cells. The high dynamic range of the EMPAD allows us to record full CBED patterns without saturation or nonlinearities, giving us access to quantitative crystallographic information. Figure 1(a) shows a virtual bright field image of the superlattice reconstructed from the scanning diffraction dataset consisted of 65,536 CBED patterns recorded in a few minutes. The symmetry maps (Fig. 1b) can be obtained by quantifying the symmetries in each CBED pattern along two mirror plane directions, $\mathrm{m} 1$ and $\mathrm{m} 2$, using correlation analysis (Fig. 1c). The polarization direction, which lies in the [112] $\mathrm{R}$ mirror plane, can thus be determined by matching with Bloch-wave CBED pattern simulations (Figs. 2a-b). Disks $\mathrm{A}$ and $\mathrm{B}$ along the polar axis show asymmetric intensities due to multiple scattering.

The intensity variations in CBED patterns is extremely sensitive to changes in diffraction conditions, i.e., excitation error $s_{g}$. We explored the effects of crystal misorientation away from the zone-axis on intensity variations in diffracted disks by simulating a series of CBED patterns for rhombohedral $R 3 c \mathrm{BiFeO}_{3}$. Figure $2(\mathrm{~g})$ shows the intensity difference between Friedel pairs A and B, a as a function of crystal misorientation, with sign reversal at a small tilt of 0.05 mrad. Thus, 4D-STEM combined with correlation analysis and Bloch-wave simulations provides a method for quantitative imaging polarization, crystal tilt and symmetry (Figs. 2c-f) in ferroelectric films at sub-nanometer spatial resolution [7].

\section{References:}

[1] RJ Zeches, et al., Science 326 (2009), p. 977.

[2] AK Yadav et al., Nature 565 (2019), p. 468.

[3] I MacLaren et al., Ultramicroscopy 154 (2015), p. 57.

[4] Z Wang et al., Phys. Rev. B 94 (2016), p. 155307. 
[5] Y-T Shao and J.-M. Zuo, Phys. Rev. Lett. 118 (2017), p. 157601.

[6] MW Tate et al., Microscopy and Microanalysis 22 (2016), p. 237.

[7] This work is supported by the AFOSR Hybrid Materials MURI, award \# FA9550-18-1-0480.

Facilities support from the National Science Foundation (DMR-1429155, DMR-1719875)

(a) Virtual BF image

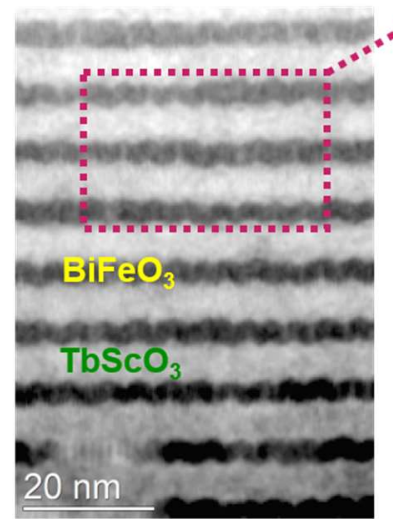

(b)

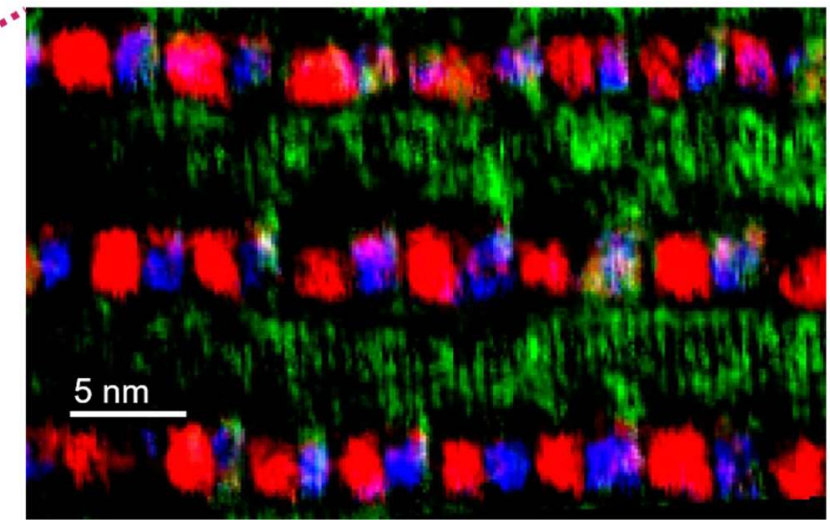

(c) Averaged CBED pattern

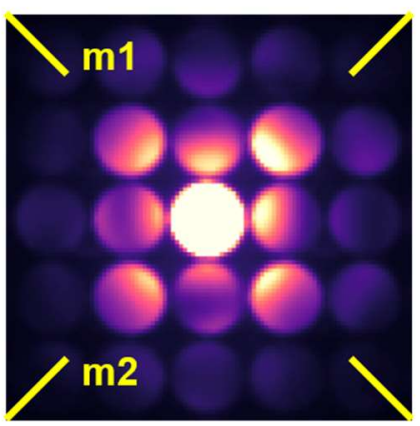

Figure 1. (a) Virtual bright field image of $\mathrm{BiFeO}_{3} / \mathrm{TbScO}_{3}$ superlattice along $[100]_{\text {pc }}$ zone axis reconstructed from the scanning CBED dataset. (b) Symmetry maps $\mathrm{m} 1$ and $\mathrm{m} 2$ can be obtained by quantifying symmetry in each CBED pattern along two mirror planes, showing alternating $109^{\circ}$ polarization domains. (c) Averaged CBED pattern of the $\mathrm{BiFeO}_{3}$ layer. Mirror plane directions $\mathrm{m} 1$ and $\mathrm{m} 2$ denote the potential projected polarization directions for rhombohedral symmetry.

(a) CBED images grouping

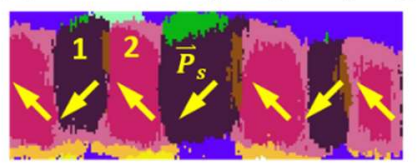

(b)

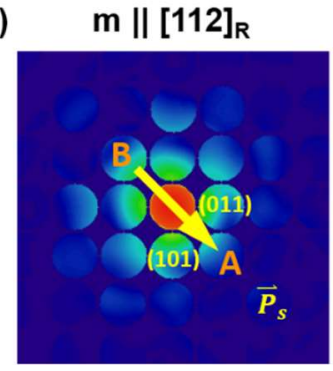

(c) Domain 1

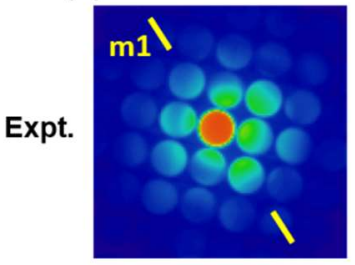

(e) Tilt $1.1 \mathrm{mrad}$

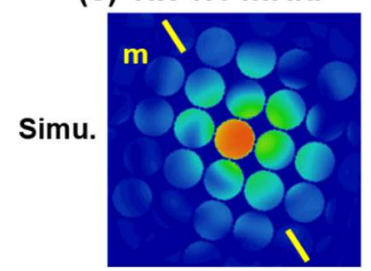

(d) Domain 2

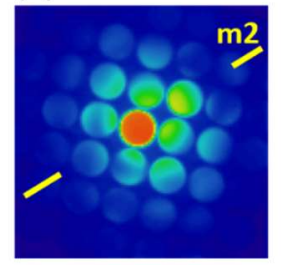

(f) Tilt $0.5 \mathrm{mrad}$

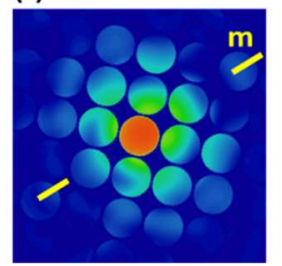

(g) Tilt vs. Disks Int. diff.

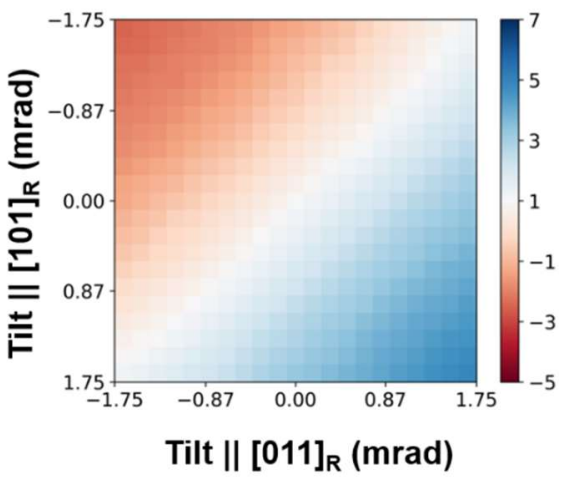

Figure 2. (a) Correlation map for CBED image stack grouping based on the correlation search algorithm. Arrows indicate direction of polarization. (b) Simulated CBED pattern of rhombohedral $\mathrm{BiFeO}_{3}$ along $[\overline{1} 11]_{\mathrm{R}}$ zone-axis using the Bloch-wave method, with high-voltage of $300 \mathrm{kV}$ and specimen thickness of 32nm. (c-d) Representative experimental CBED patterns for domains 1 and 2. (e-f) The simulated CBED patterns correspond to experimental patterns (c and d), with lattice tilted off-zone by $1.1 \mathrm{mrad}$ and 0.5 mrad, respectively. (g) Intensity difference between disks A and B as a function of small crystal tilts, showing additional causes of Friedel symmetry breaking. The difference intensities are normalized with respect to that of the CBED pattern at exact zone-axis. 